\title{
Dispositivos IoT em uma Configuração Cooperativa Fog-Cloud para suporte a Predições em um Ambiente Healthcare
}

\author{
Thiago G. Thomé ${ }^{1}$, Victor Ströele ${ }^{1}$, Mario A. R. Dantas ${ }^{1}$ \\ ${ }^{1}$ Departamento de Ciência da Computação - Universidade Federal de Juiz de Fora (UFJF) \\ - 36.036-900 - Juiz de Fora - MG - Brazil \\ \{thiagogoldoni,victor.stroele, mario.dantas\}eice.ufjf.br
}

\begin{abstract}
The world population faces an unexpected challenge with the Sars$\mathrm{CoV}-2$ pandemic and several researches are in progress to find ways to reduce contagion. This study presents a simulation effort based on changing habits and the use of wearable IoT devices to monitor and identify cases of contagion in an environment where social isolation is difficult. Thus, four scenarios with divergent prevention methods and the collected data are presented in this article, in which the environment with the most preventive measures and where the infected agents were isolated obtained the lowest contamination rate.
\end{abstract}

Resumo. A população mundial enfrenta um novo desafio com a pandemia provocada pelo Sars-CoV-2 e, por ser inesperada, diversas pesquisas estão em andamento sobre meios de reduzir o contágio. O presente estudo apresenta um esforço de simulação baseada em mudança de hábitos e no uso de dispositivos IoT vestíveis na monitoração para prevenção e identificação de casos de contágio em um ambiente onde o distanciamento social é difícil. Desta forma, quatro cenários com divergentes precauções são apresentados e os dados obtidos são apresentados neste artigo, onde o ambiente com mais medidas preventivas e no qual os agentes infectados foram isolados obteve a menor taxa de contaminação.

\section{Introdução}

Existem registros de pandemias e de seus efeitos desde o início da história da humanidade. Com a peste negra em 1343 e a gripe espanhola em 1918, a população mundial tem se adaptado à novas realidades e hábitos para superar as consequências da disseminação de novas doenças.

Com o início do surto de Covid-19 (Sars-CoV-2), especialistas e a Organização Mundial da Saúde recomendaram o aumento do isolamento social como principal medida preventiva [WHO 2020]. Porém, existem ambientes nos quais o isolamento e o distanciamento social são mais complexos, o que ocasiona em outras medidas preventivas como atenuadores no contágio. Em alguns destes ambientes, como escolas e universidades, existe a possibilidade de interrupção das atividades, entretanto em alguns outros, tais quais lares de idosos e clínicas, o isolamento social é dificultado e outras medidas complementares devem ser executadas. Como a transmissão do Covid-19 pode ocorrer durante o período de incubação, é importante um acompanhamento intensivo em pessoas que residem ou frequentem tais ambientes [Lauer et al. 2020]. Dado que o contato com 
outras pessoas não é recomendado, uma forma de proceder com o acompanhamento pode ser de maneira remota, através de dispositivos IoT.

Com a disseminação de equipamentos IoT nos últimos anos, diversos dispositivos vestíveis com sensores que permitem o monitoramento da saúde humana são facilmente encontrados no mercado. Dentre os principais sensores presentes, alguns dos mesmos podem capturar informações importantes e que podem auxiliar na detecção de uma possível contaminação. Por meio da grande quantidade de dados obtidos pelos dispositivos vestíveis, é possível que sistemas computacionais utilizem técnicas de aprendizagem para realizar predições relacionadas à saúde de uma pessoa, e com isso, auxiliar na prevenção da contaminação de um ambiente por completo.

Devido ao fato de que os dados da saúde obtidos por dispositivos IoT são dos próprios usuários e que os mesmos tendem a não tornar os próprios dados públicos, há uma grande dificuldade na busca por bases de dados que atendam à todas as características almejadas. Com isso, para concatenar o cenário do Sars-CoV-2, a IoT e os ambientes onde o distanciamento social é difícil, foi utilizado o simulador Siafu [NEC ]. Siafu é um simulador que permite controlar características de locais, comportamentos de agentes e de todo o contexto. Além de possuir características técnicas que permitem o controle total da simulação, o simulador utilizado também possui uma interface gráfica e viabiliza a criação e transferência dos dados gerados.

A aproximação dos conceitos expostos é representada através da simulação que, com comportamentos de medidas preventivas divergentes, permite a coleta dos dados da saúde dos agentes e uma análise sobre os efeitos de cada atitude tomada. Como consequência, as atitudes e comportamentos dos agentes podem ser relacionadas com as taxas de contaminação do ambiente.

Este trabalho está organizado como segue: na seção 2 explica-se os aspectos teóricos, alguns trabalhos relacionados são apresentados na seção 3, na seção 4 são descritos a proposta e o desenvolvimento. Por fim, a conclusão e trabalhos futuros na seção 5.

\section{Aspectos teóricos}

\subsection{IoT e Ambientes Domiciliares Assistidos}

A Internet das Coisas, também conhecida pela sigla "IoT" (Internet of Things), tem como conceito a conexão entre objetos físicos utilizando chips, sensores e softwares. Qualquer item que possui sensores e sistemas com intuito de operar de forma inteligente por meio de troca de informações com outros itens similares ou usuários são chamados de dispositivos IoT. Dispositivos IoT vestíveis, denominados wearables, auxiliam cada vez mais os usuários, profissionais da saúde e a telemedicina em geral. Cada vez mais frequentes nos pulsos da população, smartbands e smartwatches comumente possuem sensores capazes de obter dados como frequência cardíaca, pressão arterial, nível de oxigenação, temperatura, entre outros.

Ambientes Domiciliares Assistidos, em inglês Ambient Assisted Living (AAL), tem como base o uso de dispositivos IoT e formas de garantir que pessoas idosas ou qualquer um que precise ser assistido possam ter qualidade de vida e independência em suas residências. Acompanhado do monitoramento com wearables, o AAL se aproxima 
das pessoas com perspectivas de usabilidade e acessibilidade. Assim, grandes quantidades de dados podem ser coletadas para serem interpretadas [Nazário et al. 2017].

\subsection{Fog-Cloud}

O uso de cloud na computação consiste na possibilidade de armazenar e acessar arquivos e prover serviços através de servidores espalhados ao redor do mundo. Devido à grande quantidade de informações coletadas pelos dispositivos IoT, direcionar todo o processamento de filtragem, limpeza e aprendizagem aos serviços cloud pode ocasionar em um gargalo na efetividade do processo e um alto custo financeiro. Um ambiente fog-cloud é fundamentado na cooperação entre as edges, que são as extremidades de uma determinada região, e o cloud [Salah et al. 2020]. Em resumo, é a possibilidade de distribuir uma parte, ou todo, o processamento que seria de um cloud para diversos dispositivos de uma região. Como modelo deste ambiente cooperativo, pode-se exemplificar os edges como smartphones, computadores e servidores locais de uma residência, condomínio ou até mesmo bairros que, para remover acúmulo de processamento no cloud, exerçam serviços nos dados coletados antes de enviá-los adiante.

\section{Trabalhos relacionados}

Através do monitoramento de atividades e uso de sensores, [Silva et al. 2018] propôs o modelo HARA-RS, uma abordagem de sistema de recomendação. Com sensores no ambiente assistido e o uso de wearables, dados do acelerômetro e o posicionamento do usuário são coletados. Com as informações é possível criar um modelo da rotina de cada usuário e, caso irregularidades sejam detectadas, recomendar que o usuário busque atendimento, informar sobre a prática de hábitos sedentários, dentre outros. Uma avaliação preliminar foi realizada e os dados gerados pelo do experimento alcançaram o objetivo desejado, segundo os autores.

O estudo de [Nascimento et al. 2020] apresenta uma proposta de arquitetura baseada em simulação para o desenvolvimento de soluções com aplicações IoT. O trabalho busca permitir o desenvolvimento de simulações em diferentes contextos de cidades inteligentes e a criação de dados através do paradigma de Internet das Coisas. As simulações podem ter diversos propósitos, desde o controle de segurança pública até a saúde de pacientes contaminados, e utilizam cenários específicos, permitindo um controle total dos ambientes. Desta forma, simulações sobre a dissipação do vírus Sars-CoV-2 podem ser executadas em diferentes cenários.

Por meio da análise dos trabalhos relacionados, o presente estudo busca interligar as principais questões discutidas sobre o uso de contexto e formas de aplicação de tecnologias IoT. Junto destas características, a intenção é criar uma solução que possa auxiliar no controle da pandemia através de tecnologias eficazes de monitoramento, transmissão de dados e realização de predições.

\section{Materiais e Métodos}

Com o surto inesperado de Covid-19, o conhecimento limitado do vírus e o compartilhamento de notícias falsas, grande parte da população não teve contato com diretrizes de medidas preventivas. Com tantas informações divergentes presentes nas mídias, percebeu-se a necessidade de associar estudos mais respeitados e avançados sobre o contágio, e de forma simulada, testar os resultados obtidos até então. 
Na busca de uma possível solução foi especificado um modelo, do qual foi desenvolvido o presente trabalho, com o escopo que engloba desde os hábitos dos agentes da simulação até o ambiente computacional responsável por realizar as predições em healthcare. O presente modelo de simulação proposto é representado na Figura 1.
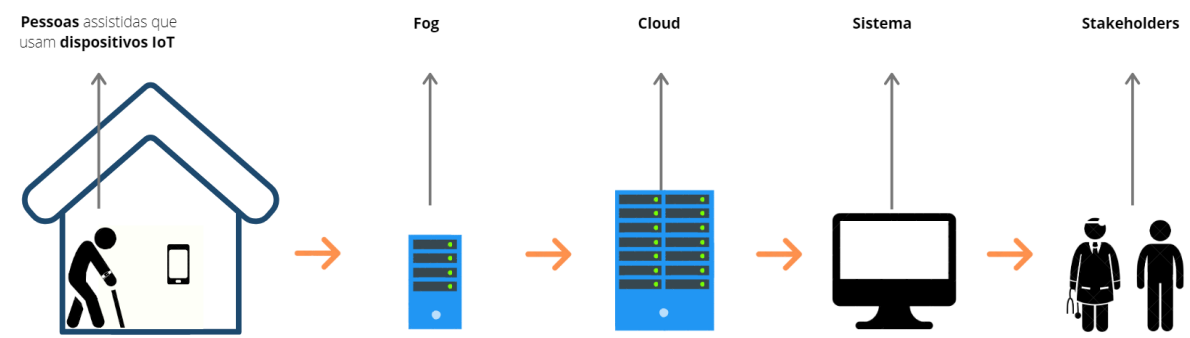

Figura 1. Esboço do modelo proposto

Na solução proposta, os agentes são as pessoas que necessitam de acompanhamento e utilizam dispositivos IoT vestíveis em um ambiente domiciliar assistido. As fogs são dispositivos que correspondem a uma determinada região de agentes e que executam processos computacionais nas informações recebidas através dos agentes. A cloud é responsável por receber informações previamente processadas de diversas fogs e efetuar rotinas de aprendizagem. O sistema representa uma aplicação web que acessa a cloud e onde predições, gráficos e relatórios podem ser acessados. Por fim, os stakeholders são os usuários do sistema que tem interesse na informação gerada, eles podem ser parentes ou profissionais da saúde responsáveis pelos pacientes.

Para uma avaliação preliminar, somente a simulação através do Siafu e a criação dos dados coletados pelos wearables foram implementados. No ambiente simulado o distanciamento social é complexo, que neste modelo, é um lar de idosos com 18 agentes. O ambiente é composto por seis dormitórios, dois banheiros, uma sala de convivência e um refeitório. $\mathrm{O}$ ambiente descrito é apresentado pela Figura 2.

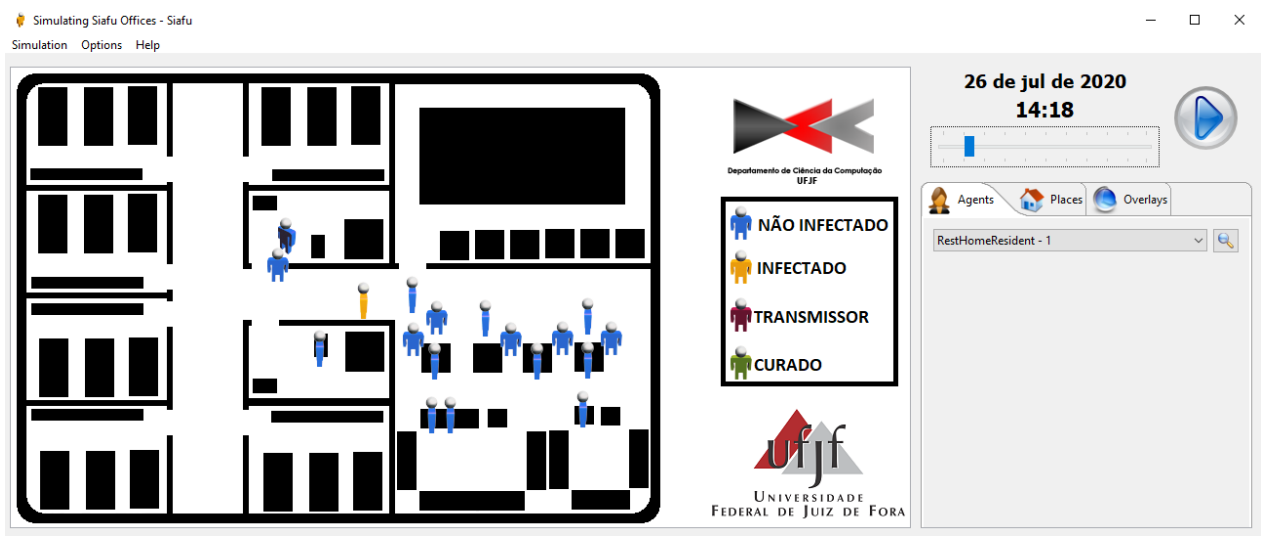

Figura 2. Ambiente simulado

Para expressar a fidelidade do modelo simulado, diversas características personalizadas foram acrescentadas aos agentes. Cada um possui horário para acordar e dormir, intervalos de ida ao banheiro e comportamento com as medidas preventivas antes e depois da revelação do primeiro caso no ambiente. Com o avanço exponencial de casos, é 
comum que a população só comece a mudar seus hábitos quando alguém próximo seja diagnosticado e, por isso, a simulação permite parametrizar tais características.

Com a recente pandemia de Covid-19, muitos pesquisadores ao redor do mundo iniciaram estudos sobre os aspectos da nova doença. Devido a diferentes formas de como o combate é feito em cada região e país, alguns números como porcentagem de assintomáticos são relativamente divergentes. Para a simulação foi necessário realizar uma compilação de estudos mais conceituados e recentes.

Com os estudos de [Liu et al. 2020], [Lauer et al. 2020] e [Ferguson et al. 2020], a taxa de assintomáticos para a simulação foi ajustada para $30 \%$, ou seja, a cada dez pessoas contaminadas, três serão transmissores e os dados capturados por wearables não apresentarão alterações, impedindo a identificação do indivíduo.

De acordo com [Chu et al. 2020], em sua revisão sistemática e metanálise, o uso de máscaras N95, classificadas como PFF2 no Brasil, ou até de outras máscaras pode reduzir a chance de contágio de forma significativa. Desde que utilizadas de forma correta, os estudos indicam que as máscaras PFF2 podem reduzir em até 95\% a chance de contágio, enquanto máscaras de algodão podem alcançar $67 \%$.

Outra medida preventiva analisada foi o isolamento social, que para uma distância de $1 \mathrm{~m}$ apresenta diminuição das chances de contágio em $82 \%$ e para cada metro adicional os riscos caem pela metade. De forma análoga, outra forma de proteção é o uso de protetores de olhos, que também apresenta uma redução significativa, alcançando valores de $78 \%$ de atenuação dos riscos.

O modelo de simulação proposto permite a comparação entre diversas situações, desde testes sobre a eficácia de métodos de prevenção até a forma de como a mudança de comportamento dos agentes influencia na contaminação de um ambiente. Com isso, quatro ambientes foram preparados para permitir a comparação sobre os hábitos e as práticas dos agentes como descrito na tabela 1.

Tabela 1. Parâmetros utilizados nas simulações

\begin{tabular}{|l|r|l|l|l|}
\hline \multirow{2}{*}{ Hábitos } & \multicolumn{3}{|c|}{ Ambiente simulado 1 e 3 } & \multicolumn{2}{c|}{ Ambiente simulado 2 e 4 } \\
\cline { 2 - 6 } & $\begin{array}{l}\text { Hábitos antes da } \\
\text { divulgação do } \\
\text { primeiro caso no } \\
\text { ambiente }\end{array}$ & $\begin{array}{l}\text { Hábitos depois da } \\
\text { divulgação do } \\
\text { primeiro caso no } \\
\text { ambiente }\end{array}$ & $\begin{array}{l}\text { Hábitos antes da } \\
\text { divulgação do } \\
\text { primeiro caso no } \\
\text { ambiente }\end{array}$ & $\begin{array}{l}\text { Hábitos depois da } \\
\text { divulgação do } \\
\text { primeiro caso no } \\
\text { ambiente }\end{array}$ \\
\hline Uso de máscara PFF2/N95 & $0 \%$ & $100 \%$ & $90 \%$ & $10 \%$ \\
\hline Uso de outras máscaras & $50 \%$ & $0 \%$ & $50 \%$ & $0 \%$ \\
\hline Uso de protetor de olhos & $0 \%$ & $90 \%$ & $1,0 \mathrm{~m}$ & $1,5 \mathrm{~m}$ \\
\hline Aumento médio da distância & $0 \mathrm{~m}$ & $1,5 \mathrm{~m}$ & $00 \%$ \\
\hline
\end{tabular}

Nos ambientes simulados 1 e 3 , os agentes pouco mudaram seus hábitos após o início da pandemia, com somente 50\% utilizando máscaras antes do primeiro caso no ambiente. Tanto o uso de máscaras PFF2, uso de protetor de olhos e o hábito de aumentar a distância foram ignorados pelos mesmos.

Em contrapartida, todos os agentes dos ambientes simulados 2 e 4 utilizaram máscaras PFF2 ou outras após o começo da doença, respectivamente $90 \%$ e $10 \%$. Além das máscaras, é importante notar o uso do protetor de olhos por $50 \%$ da população e o 
aumento médio da distância em um metro.

Como complemento à precisão da simulação, nos ambientes 1 e 2 quando algum agente contaminado atinge um quadro clínico com sintomas, o mesmo é afastado da simulação. Em compensação, os agentes dos ambientes 3 e 4 não são isolados quando apresentam os primeiros sintomas. Assim, não há contaminação por parte de um agente no qual a doença foi detectada nos dois primeiros ambientes, impedindo que outros agentes continuem suas rotinas diárias com um agente contaminado no qual todos saibam de sua situação.

Em todos os modelos simulados, os hábitos dos agentes sobre afastamento e equipamentos de proteção foram similares após a descoberta da contaminação no ambiente. Com isso, a diversidade e a variedade entre as simulações se encontram sobre as medidas preventivas e o afastamento de agentes com sintomas. Os propósitos para tais fundamentos é de entender e demonstrar as consequências da disseminação de um vírus onde a transmissão tem início durante o período de incubação e das diferentes formas de prevenção aplicadas[Lauer et al. 2020].

\section{Resultados}

Ao longo do período de teste, foram efetuadas 50 execuções para cada ambiente simulado. Em todos os experimentos os agentes se comportaram de acordo com os parâmetros definidos e os valores obtidos dos sensores foram capturados. Os pontos de interesse do estudo estão descritos na tabela 2, onde os valores médios são expostos e os efeitos da disseminação da doença no ambiente são as consequências dos hábitos preventivos e do isolamento.

Tabela 2. Valores médios obtidos nas simulações

\begin{tabular}{|l|r|r|r|r|}
\hline \multirow{2}{*}{ Situação } & \multicolumn{3}{|c|}{ Agentes contaminados } \\
\cline { 2 - 5 } & Ambiente simulado 1 & Ambiente simulado 2 & Ambiente simulado 3 & Ambiente simulado 4 \\
\hline Contaminados até a identificação do $1^{\mathrm{a}}$ caso & $65,22 \%$ & $11,44 \%$ & $61,56 \%$ & $11,00 \%$ \\
\hline Contaminados após a identificação do $1^{\circ}$ caso & $2,67 \%$ & $0,67 \%$ & $15,44 \%$ & $14,89 \%$ \\
\hline Contaminados até o final da simulação & $67,89 \%$ & $12,11 \%$ & $77,00 \%$ & $25,89 \%$ \\
\hline
\end{tabular}

Com os resultados das execuções, foi detectado que em torno de $67,89 \%$ dos agentes do ambiente simulado 1 foram contaminados até o final da simulação. Do total de infectados, 65,22\% foram contaminados antes da constatação do primeiro caso. De outra forma, apenas $12,11 \%$ dos agentes do ambiente simulado 2 foram contaminados e, sendo destes, somente $0,67 \%$ foram contaminados após a identificação do primeiro caso no ambiente.

Em compensação, quando não houve o isolamento dos agentes com sintomas, como nas simulações 3 e 4 , as quantidades de contaminações foram relativamente superiores quando comparadas com os dois primeiros ambientes. Com isso, 77,00\% dos agentes foram contaminados no ambiente simulado 3 e 25,89\% no ambiente 4 .

Na figura 3, em decorrência das contaminações ocorridas, foi possível analisar a grande concentração de contaminações durante a primeira semana nos ambientes simulados 1 e 3 . Além do fato de que, mesmo com um menor número de contaminados, uma distribuição mais equivalente nos ambientes 2 e 4. 


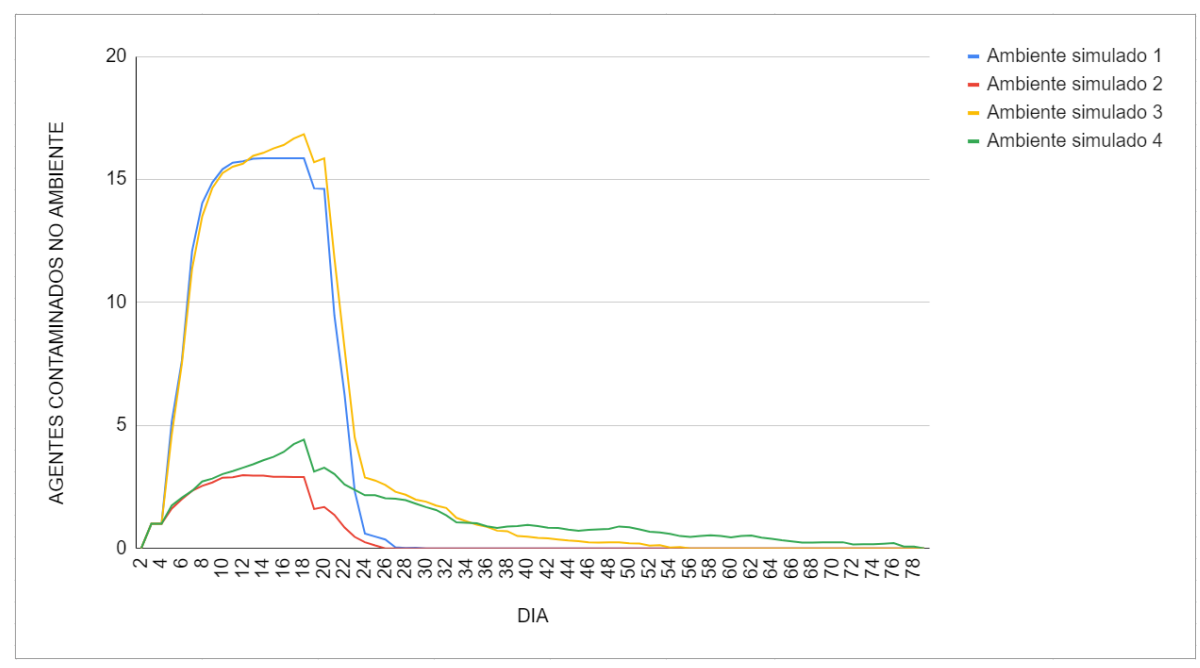

Figura 3. Agentes contaminados x dia após o início da contaminação

Os dados coletados com o resumo diário para cada ambiente e os arquivos executáveis de cada simulação estão disponíveis no endereço eletrônico: https://github.com/thiagogoldoni/WSCAD-WIC2020

\subsection{Discussões}

Diferentes formas de análise podem ser realizadas com os resultados obtidos. Sendo feita uma observação nos ambientes 1 e 2, mesmo com diferentes hábitos de prevenção, a prática do isolamento faz com que as contaminações nos ambientes sejam encerradas antes do trigésimo dia, que são resultados significativamente melhores quando comparados com os do ambiente 3 e 4, que estão após o quinquagésimo dia. Outra forma de observação dos resultados é quando uma comparação entre os ambientes 1 e 3 é feita com os ambientes 2 e 4 . A quantidade média de agentes contaminados ao mesmo tempo nas simulações 1 e 3 alcançam valores até cinco vezes maiores que nos ambientes simulados 2 e 4. Tal resultado ressalta a necessidade do isolamento de agentes contaminados logo após a identificação.

Através das conferências realizadas, o ambiente simulado 2 com o uso das máscaras e protetor de olhos, aumento da distância e isolamento dos infectados apresentou os melhores resultados quando comparado com os demais. Com menos agentes infectados, é possível perceber a importância das medidas de prevenção no controle da disseminação do Sars-CoV-2 em ambientes onde o isolamento social é difícil.

\section{Considerações finais}

Com a avaliação preliminar do modelo e dos ambientes simulados, foi possível não só identificar a importância do isolamento e de hábitos preventivos após a constatação do primeiro caso mas, principalmente, a relevância destas mesmas atitudes antes do conhecimento da existência de algum agente contaminado para facilitar a contenção dos casos.

Para trabalhos futuros, pretende-se implementar um ambiente cooperativo fogcloud com para filtrar, limpar e realizar rotinas de aprendizagem sobre os dados e, como complemento, o desenvolvimento do sistema web que permita o acesso e consumo dos dados. 
Agradecimentos à Professora Dra. Hélady Sanders Pinheiro, do programa de pós-graduação em saúde da UFJF, pela colaboração nesta pesquisa. Reconhecemos a importância dos apoios dos projetos SIGOM do INESC P\&D e RED da Petrobrás em cooperação com a UFJF.

\section{Referências}

Chu, D. K., Akl, E. A., Duda, S., Solo, K., Yaacoub, S., and Schünemann, H. J. (2020). Physical distancing, face masks, and eye protection to prevent person-to-person transmission of sars-cov-2 and covid-19: a systematic review and meta-analysis. volume 395, pages 1973-1987. The Lancet.

Ferguson, N. M., Laydon, D., Nedjati-Gilani, G., Imai, N., Ainslie, K., Baguelin, M., Bhatia, S., Boonyasiri, A., Cucunubá, Z., Cuomo-Dannenburg, G., Dighe, A., Dorigatti, I., Fu, H., Gaythorpe, K., Green, W., Hamlet, A., Hinsley, W., Okell, L. C., van Elsland, S., Thompson, H., Verity, R., Volz, E., Wang, H., Wang, Y., Walker, P. G., Walters, C., Winskill, P., Whittaker, C., Donnelly, C. A., Riley, S., and Ghani, A. C. (2020). Impact of non-pharmaceutical interventions (npis) to reduce covid-19 mortality and healthcare demand. Imperial College London.

Lauer, S. A., Grantz, K. H., Bi, Q., Jones, F. K., Zheng, Q., Meredith, H. R., Azman, A. S., Reich, N. G., and Lessler, J. (2020). The incubation period of coronavirus disease 2019 (covid-19) from publicly reported confirmed cases: Estimation and application. volume 172, pages 577-582. Annals of Internal Medicine.

Liu, Y., Yan, L. M., Wan, L., Xiang, T. X., Le, A., Liu, J. M., Peiris, M., Poon, L., and Zhang, W. (2020). Viral dynamics in mild and severe cases of covid-19. pages 656-657. Lancet Infect Dis.

Nascimento, M. G., Braga, R. M. M., David, J. M. N., Dantas, M. A. R., and Colugnati, F. A. B. (2020). Covid-19: A simulation-based architecture proposal for iot application development. In International Conference on High Performance Computing \& Simulation. HPCS. (Submetido).

Nazário, D. C., Campos, P. J., Inacio, E. C., and Dantas, M. A. (2017). Quality of context evaluating approach in aal environment using iot technology. pages 558-563. CBMS 2017.

NEC. Siafu an open source context simulator. http://siafusimulator.org/ (acesso em 07/2020).

Salah, F. A., Desprez, F., and Lebre, A. (2020). An overview of service placement problem in fog and edge computing. volume 53. Association for Computing Machinery.

Silva, G. D. I., Stroele, V., and Dantas, M. A. R. (2018). Sistema de recomendação para a saúde baseado em computação ubíqua. In Workshop de Iniciação Científica em Arquitetura de Computadores e Computação de Alto Desempenho, pages 502-507. WSCAD-WIC 2018.

WHO (2020). Coronavirus disease (covid-19) advice for the public. https://www.who.int/emergencies/diseases/novel-coronavirus-2019/advice-for-public/ (acesso em 07/2020). 